\title{
Comparison of Efficacy of Three Ni-Ti Instruments in Removal of Gutta-Percha from Root Canal during Retreatment- An In Vitro Study
}

\author{
Dr. Anil.K.Tomer ${ }^{1}$, Dr. Anjali Miglani ${ }^{2}$, Dr. Smita Sahni ${ }^{3}$, Dr. B. Vinay Goud ${ }^{4}$, \\ Dr. Sneha Vaidya ${ }^{5}$, Dr. Anamika Kumari ${ }^{6}$. \\ 1,2,3,4,5,6(Conservative Dentistry And Endodontics, DivyajyotiCollegeOf Dental Sciences And Research, India)
}

\begin{abstract}
Aim:This study compares the efficacy of three different retreatment file systems and determines which retreatment file requires less time in removal of previous root filling material.

Methodology- Thirty anterior teeth with single root canal were used in this study.The teeth were randomly divided into three experimental groups of ten specimens each.They were instrumented and obturated using mono -cone technique with gutta-percha $(G P)$ and sealer.

Removal of gutta-percha was performed with the following devices and techniques: EDGEFILE XR, MTWO, and R-ENDO. For all cases, the following data were recorded: procedural errors, duration of retreatment and canal wall cleanliness which was evaluated usingstereomicroscope at $6 X$ magnification. Photographs were taken for further analysis using computer image analysis program.ANOVA test and Bonferroni multiple comparison tests were used for statistical analysis.

Results: No system completely removed the root filling material from root canal walls.EdgeFile XR retreatment files removed the maximum amount of filling material from the canal walls. The mean operating time was minimum with Mtwo group files.

It was concluded that EDGEFILE XR, rotary retreatment system proved to be the most efficient method of removing gutta-percha and sealer in comparison to the other two retreatment files and M-TWO retreatment files required less time to remove root filling material than the other instruments.
\end{abstract}

Keywords:EdgeEndoFiles, GuttaPercha, MTwoFiles, R-Endo files, Retreatment, Stereomicroscope.

\section{Introduction}

Quality of dental care provided to the general population has improved immensely due to advancement in technology. These advancements along with increased dental patient education and awareness, have ensured that the dentition continue to remain a key part of people's lives. With increased population life span, the need to maintain a dentition for a prolonged period of time has led to a series of advanced dental procedures that were nonexistent few years ago. Therefore the need for conventional root canal therapy has also increased substantially1. Root canal treatment has reported a success rate between $62 \%$ and $96 \%$ over the last few decades2. However it is unfortunate that a certain number of endodontically treated teeth have to be retreated. Variety of reasons have been attributed for the failure of root canal treatment, such as poorly treated and obturated canals, complications with respect to instrumentation, over extensions of obturatingmaterialsand complicated root canal anatomy3.

In 1986, late Dr Herbert Schilderquoted the term "RETREATODONTICS" and said that thefuture of endodontics lies in the "Retreatment of Endodontic Failures". When root canal therapy fails, treatmentoptions include conventional retreatment, periradicularsurgery or extraction. Whenever possible,the retreatment option is preferred because it isthe most conservative method to solve theproblem.[2]

The main goals of orthograde retreatment areregaining access to the apical foramen by complete removal of root canal filling materials thusfacilitating sufficient cleaning and shaping of the complete root canal system and final obturation.[3,4] Necrotic tissue or bacteria, covered by remaining GP [GuttaPercha] or sealer may be responsible for periapical inflammation or pain. $\mathrm{E}$ faecalis is the main species found in cases of rootfilled teeth associated with peri-radicular lesions.[1,5,6]

Only if the filling material can be removed completely and the root canal negotiated to the apical foramen, allowing thorough debridement, can the prerequisites for a successful retreatment be fulfilled. [1,4]. Several techniques have been proposed to removefilling materials from the root canal system, includingthe use of endodontic hand files, Nickel-Titanium (Ni-Ti)rotary instruments (Masiero\& Barletta 2005, Hammadet al. 2008), Gates Glidden burs, heat, ultrasonicinstruments (Wilcox 1989), laser (Viducic et al.2003) and use of adjunctive solvents. Conventionally, the removal of gutta-percha using hand files with orwithout solvent can be 
a tedious, time-consuming process especially when the root filling material is well compacted (de Oliveira et al. 2006). Therefore, the useofNiTi rotary instruments in root canal retreatmentmight decrease patient and operator fatigue (Tasdemiret al. 2008a).

Three NiTi systems have recently been introduced: R-Endo (Micro-Mega, Besancon, France), Mtwo (Sweden \& Martina, Padova, Italy) andEdgefileXR(EdgeEndo, USA).

$\mathrm{R}$-Endo retreatment files were used in a gentlein and out motion on the canal walls according tomanufacturer's instruction. A manual file Rm wasused first to relocate the canal orifices, then the $\operatorname{Re}(\operatorname{size} 25$, 0.12 taper) instrument removed the first $2-3 \mathrm{~mm}$ of the filling. R1 (size 25, 0.08 taper) andR2 (size 25, 0.06 taper) were used to one-third andtwo-thirds of the estimated working lengthrespectively. Finally R3 (size 25, 0.04 taper) wasused at the working length to complete the removalof filling material from the canal.Mtwo Retreatment Files consist of two instrumentswith active cutting tip: R1 (size 25, 0.05 taper) and R2(size 15, 0.05 taper) (Somma et al. 2008, Tasdemiret al. 2008a). They have an S-shaped cross-section asdo the files of the basic sequence, but a shorter pitchlength to enhance the advancement of the file into thefilling material. These instruments are characterized bytwo cutting edges, which are claimed to cut dentineffectively (Gergi\&Sabbagh 2007).

Recently, EdgeFile XR retreatment nickel-titanium (Ni-Ti) rotary files that are made of an annealedheat- treated $\mathrm{Ni}^{-} \mathrm{Ti}$ alloy brand named Fire- WireTM,have been introduced to the market. The deformation and strength characteristics of metals and metal alloys could be changed with heat treatment. According to manufacturer Fire- Wire ${ }^{\mathrm{TM}} \mathrm{Ni}-\mathrm{Ti}$ yields performance- enhancing durability that provides incredible flexibility, so that XR files will enhance and expedite the endodontic retreatment. The cyclic fatigue has been custom tested and found to be twice that of the other file systems. System includes four files - R1 (25/0.12), R2 (25/0.08), R3 (25/0.06), R4 (25/0.04) - that are used in crown- down manner. All files have constant taper and parabolic cross section.

\section{II.Materialsand Methods}

Selection of teeth: thirty maxillary central and lateral incisors with mature root apices and single canal extracted for periodontal reasons were used. Teeth with root caries, cracks on the root surface, curved roots and extremely calcified canals were excluded. Soft tissue and calculus were removed mechanically from the root surface.

\section{Initial root canal treatment}

Each tooth was decoronated at the cemento-enameljunction (CEJ) with a diamond disc to facilitate straight line access for instrumentation and obturation. Proper access was established and the apical patency was determined by inserting an ISO\# $10 \mathrm{~K}$-file until it appeared at the apical foramen. Working length was determined by placing a size $15 \mathrm{~K}$-file into the canal until it appeared at the apical foramen; this length was measured and the working length was set $0.5 \mathrm{~mm}$ short of this distance. A circumferential 'staging platform' was established near the canal orifice, ensuring a uniform workinglength (WL) of $15 \mathrm{~mm}$ in each tooth.

Cleaning and shaping were performed using a modified step-back flare technique. The coronal third was flared with sizes 1-3 Gates Glidden drills (DentsplyMaillefer). Canal preparation was carried out by the sequential use of K-files (DentsplyMaillefer, Ballaigues, Switzerland) up to size 30 at working length; a stepback procedure in $1 \mathrm{~mm}$ increments to a file size 50 was then carried out. Upon withdrawal of each instrument, canals were irrigated alternatively with $5.25 \%$ sodium hypochlorite $(\mathrm{NaOCl})$ and $17 \%$ ethylenediaminetetraaceticacid (EDTA).

\section{Root canal obturation:}

The root canal of each tooth was dried with paper points and obturated with gutta-percha (GP) and sealapexusing a cold lateral compaction technique. The coronal access cavities of the specimens were sealed with temporary filling material (Cavit, DeTreyDentsply). The quality of the root fillings was confirmed using postoperative radiographs. All teeth were stored at room temperature for 30 days to allow complete setting of the sealer.

\section{Retreatment Techniques:}

All the specimens were randomly divided into three experimental groups $(n=3)$ with 10 specimenseach for removal of gutta-percha by using one ofthe following techniques:

Group A:EdgeFile XR retreatment files

Group B: R- Endo

Group C: M-two retreatment files 
All instruments were used in a crown-down technique on a low-torque rotary engine driven motor (XSmart; DentsplyMaillefer) in the preset torque levels recommended by the manufacturer for each type of instrument, and at a constant speed of $500 \mathrm{rpm}$.

\section{GroupA:EdgeFileXR}

R1 (25/0.12), R2 (25/0.08), R3 (25/0.06) and R4 (25/0.04) files were used in crown- down manner with light to medium pressure in apical way, respectively. The sequence was repeated until R4 reaches to WL. Final apical preparation was then performed using the EdgeFile X3- C4 file (size 40/0.06 taper). As a safety feature the files are designed to unwind. They may be used until the files unwind backwards.

\section{Group B:R-Endo}

R-Endo rotary system was used as recommended by manufacturer in crown-down technique and pushand retains motion circumferentially. R1, R2 and R3 were used respectively to remove the root filling material from the coronal, middle and apical thirds. The removal of the obturation material was considered complete when the working length was reached, no material was observed between the flutes of the files, and the walls of the canal were smooth and free of visible debris.

\section{Group C:M-two}

Retreatment Files (M-two R) were also used according to the instructions of the manufacturer. Root canals were instrumented in a simultaneous technique to the working length using M-two R2 (size 25, 0.05 taper) in a brushing action with lateral pressing movements. Progression of the rotary file was performed by applying slight apical pressure and frequently removing the files to inspect the blades and clean the debris from the flutes. Lastly, conventional Mtwo rotary instrument (size 30, 0.05 taper) was used at WL.

\section{Evaluation}

a) Remaining gutta-percha and sealer

All specimens were rendered transparent according to the following technique described by Schirrmeister et al. 17. The specimens were decalcified in 5\% nitric acid for 72hours, washed for 4 hours and dehydrated in increasing concentrations of alcohol (80\%, for 12 hours, $90 \%$ for 1 hours and $99 \%$ for 3 hours). The roots were cleared subsequently using methysalicylate. The GP/sealer remnants on the canal walls were imaged on a black background in mesio-distal (M-D) direction using a stereomicroscope at 6Xmagnification. Each canal was divided into coronal, middle and apical thirds from the 'staging platform' to the terminus of the apical preparation. The area of GP/sealer remnants as well as the canal wall was measured using image analyzer software.

\section{b) Operating time}

The operating time which elapsed from initial GP removal with the first instrument until reaching the original working length was recorded as T1. The time required to achieve satisfactory GP removal after reaching the working length was recorded as T2. Total time for treatment was the sum of T1 and T2.

\section{Statistical Analysis}

Analysis of variance (ANOVA) was used to analyze the differences in the percentages of GP/ sealer remnants covered area amongst the four groups. One-way ANOVA was applied to compare the operating time amongst the four groups. Bonferroni test was performed as the post hoc multiple comparison method.

\section{a) Remnants of material}

\section{Results}

All instruments left filling material inside the root canal. The specimens retreated with the EdgeFile XR left less filling material inside the root canals than other groups but significance difference was found betweenEdgeFile XR and M-Two, R-Endo and Mtwo $(<0.001)$. (Table I) The comparison of GP/Sealer remnants at different levels among three groups using Bonferroni Method test showed that difference in different levels was found to be maximum between coronaland apical levels (4.062) while it was minimum between coronal and middle levels (1.751).

Table I - Area fraction of root canal wall covered by GP/sealer remnants after retreatmentM-D direction

\begin{tabular}{|l|l|l|l|l|l|l|l|}
\hline \multicolumn{2}{|l|}{} & \multicolumn{2}{|l|}{} & & \\
\hline & Group & Mean & SD & Mean & Standard Deviation & Mean & $\begin{array}{l}\text { Standard } \\
\text { Deviation }\end{array}$ \\
\hline & A (Edgefile-XR) & 3.24 & 1.29 & 5.17 & 1.81 & 7.51 & 2.51 \\
\hline & B(R-Endo) & 3.46 & 1.06 & 5.15 & 1.58 & 7.54 & 2.04 \\
\hline & C (M-Two) & 4.42 & 1.19 & 6.60 & 1.34 & 11.01 & 2.33 \\
\hline
\end{tabular}


Comparison of efficacy of three Ni-Ti instruments in removal of gutta-percha from root canal

\begin{tabular}{|l|l|l|l|}
\hline & 8.054 & 6.732 & 10.651 \\
\hline & $<0.001$ & 0.001 & $<0.001$ \\
\hline
\end{tabular}

\section{b) Operating time}

The mean time taken for complete procedure was found to be minimum in Group C $(5.08 \pm 0.64 \mathrm{~min})$ while it was found to be maximum in Group B $(7.93 \pm 1.03 \mathrm{~min})$. (Table II) The efficacy of groups in terms of mean time taken to complete the procedure was:

\section{M-Two > Edge File XR R-Endo}

Table II-Time taken for complete procedure in different groups.

\begin{tabular}{|l|l|l|l||l|l|l|l|l||}
\hline \multirow{2}{*}{ Group } & $\begin{array}{l}\text { No. of } \\
\text { samples }\end{array}$ & $\begin{array}{l}\text { Mean } \\
\text { time }\end{array}$ & \multicolumn{2}{|c|}{ Range } & Max & F(Analysis & $\begin{array}{l}\text { P (Level of } \\
\text { of Variance) } \\
\text { significance) }\end{array}$ \\
\hline & $\begin{array}{l}\text { A (Edgefile- } \\
\text { XR) }\end{array}$ & 10 & 6.10 & 1.00 & 3.58 & 7.45 & 21.350 & $<0.001$ \\
\hline & B (R-Endo) & 10 & 6.41 & 1.21 & 4.07 & 8.81 & & \\
\hline & C (M-Two) & 10 & 5.08 & 0.64 & 3.49 & 5.72 & & \\
\hline
\end{tabular}

\section{Discussion}

The primary reason for a negative outcome following the root canal treatment is the persistence of bacteria within the intricacies of the root canal system.18 Complete removal of pre-existing filling material from canals is a prerequisite for successful nonsurgical root canal retreatment.19 This procedure can uncover residual necrotic tissues or bacteria that may be responsible for persistentperiapical inflammation, and allow further cleaningand refilling of the root canal system.20

In the present study, the teeth were decoronated to ensure standardization of specimens by eliminating some variables, such as anatomy of the dental crown and access to root canals thereby allowing more reliable comparison between the proposed retreatment techniques. .Different methodologies have been reported to evaluate the amount of filling material remaining inside the canal after retreatment procedure. It can be assessed radiographically 21 , roots can be split longitudinally and remaining gutta-percha and sealer were measured linearly or using scoring system 22 or making the teeth transparent17. In addition computer tomography 23 and operating microscopes 20 have also been used for this purpose. Ideally, three-dimensional visualization of the root canal system would provide a better understanding of the distribution of the debris after retreatment.16,24 Amongst them, the transparent teeth method is cost-effective and sensitive enough to identify small area of residual GP/sealer on the canal wall.20, 25 In the present study, the roots were made transparent to allow measurement of the area of residual filling material.

For the present study xylene was selected from a variety of different solvents which are recommended for endodontic retreatment which includes eucalyptol, halothane, methyl chloroform, chloroform, turpentine. 14 Xylene slowly dissolves the gutta-percha, thus allowing better control and removal of softened gutta-percha rather thanliquefied gutta-percha.26 Although chloroform has been shown to be most effective gutta-percha solvent when compared with other solvents but its use is controversial.27 It has been reported to be locally toxic when in contact with perirdiculartissues. Additionally it is hepatotoxic and nephrotoxic and is classified as a carcinogenen.22, 28.

Different methods have been applied to remove root canal filling material from canals. These include use of hand files, ultrasonic files, engine driven instruments and lasers.3, 14, 15 Conventionally, the removal of gutta-percha using hand files with or without solvent can be a tedious and time consuming process, especially when the root filling material is well condensed.16 Therefore the use of rotaryNiTi instruments in root canal retreatment might decrease patient and operator fatigue. In the current study, all retreatment techniques left GP/sealer remnants within the root canal. This finding confirms previous results reported by numerous investigators using different retreatment instruments, techniques and solvents.3, 13, 16 Furthermore, the present investigation showed that that rotaryNiTi instruments, the EdgeFile XR instrumentation was significantly more effective than Mtwo and R-Endo group in terms of residual material, whereas no statistical difference was found amongst the EdgeFileXR and R-Endo instrumentation group. There have not been sufficient studies comparing the efficiency of the EdgefileXRto other retreatment files conventionally used for retreatment in endodontics. In the present study the better performance of EdgefileXRinstruments may be because they are made of an annealed heat- treated $\mathrm{Ni}-\mathrm{Ti}$ alloy brand named Fire- Wire ${ }^{\mathrm{TM}}$.The heat treated Fire-Wire ${ }^{\mathrm{TM}} \mathrm{Ni}-\mathrm{Ti}$ yields performance enhancing durability (PED) that provides not only incredible flexibility, but according to the manufacturers it performs much better than other files in cyclic fatigue testing; a key indicator of file strength and durability.Edgefile XR enhances and expedite the re-treatment of a root canal.Parabolic Cross-Section 
combines the attributes of being a highly efficient and flexible instrument while being extremely safe and resistant to fracture.According to the present study system,R-Endo left almost similar amount of filling material in the canal walls compared withEdgefileXR. Tasdemir et al.16 reported that ProTaper, R-Endo and manual instrumentation groups have similar effectiveness in removing filling material in straight root canals.In the present study significant difference was found between R-Endo group and Mtwo, this is in accordance to the study done by Tasdemir et al.16 The Mtwo instruments have an S-shaped cross-sectioned, an increasing pitch length in the apical-coronal direction which is characterized by positive rake angle with two cutting edges, which are claimed to cut dentin effectively.29 Unlike other NiTiinstruments, theMtwo rotary instruments do not require a crown-down instrumentation sequence. Using the Mtwo instruments with the single length preparation leaves more filling material in the canal during retreatment.

Advantages of rotary files includes maintenance of canal shape, reduced working time and reduced operator fatigue whereas disadvantages includes higher incidence of file separation [28,10], extrusion of obturating material and debris through the apical foramen, alterations of root canal morphology [10], but this study utilized the parameter which is time required for retreatment procedure as criteria for evaluation. It was concluded in this study that rotary instruments were significantly fast in removing gutta-percha, while Mtwo require less time than EdgefileXR and R-Endo rotary instruments. This is due to the specific design of M-two, resulting in aggressive cutting edges and positive rake angle which require less energy to cut dentin. The number of instruments in EdgefileXR and R-Endo also affect the working time even though they are more effective in removal of guttapercha.

\section{VI.Conclusion}

Within the parameters of this study, the following conclusions may be drawn:

$>$ None of the techniques removed all filling materials from root canal walls.

$>$ EdgefileXRre-treatment files and R- Endo re-treatment files left significantly less gutta-percha and sealer than M-two system

$>$ Re-treatment with M-two system Ni-Ti rotary systems was significantly faster compared to other two rotary techniques used in the removal of gutta-percha/sealer.

\section{References}

[1]. Ralan Wong, Conventional Endodontic Failure And Retreatment, Dental Clinic Of North America,Modern Endodontic Practice.

[2]. Advanced Endodontics, Clinical retreatment and surgery, John S Rhodes.

[3]. Cohen's Pathways of the Pulp Expert Consult, 10th Edition.

[4]. Stabholz A and Friedman S. Endodontic Retreatment-Case Selection and Technique. Part 2: Treatment Planning for Retreatment. J Endod 1988;14:607-14.

[5]. 5.Hulsmann M and Stotz S. Efficacy, cleaning ability and safety of different devices for GP removal in root canal retreatment. IntEndod J 1997;30:227-33.

[6]. 6.Lovdahl PE. Endodontic Retreatment. Dent Clin North Am 1992;36:473-90.

[7]. 7. Ingle JI, Bakland LK, Baumgartner CJ. Ingles Endodontics 6.6th edition. Hamilton: BC Decker Inc;2008.

[8]. 8. Rocas IN, Jung II-Y, Lee CY, Siqueira JF. Polymerase chain reaction identification of microorganisms in previously root filled teeth in a South Korean population. J Endod 2004;30,7:504-8.

[9]. 9.Cohen S and Hargreaves KM. Pathways of the Pulp. 9th ed. St. Louis: Mosby; 2006.

[10]. 10.Schirrmeister JF, Meyer KM, Hermanns P, Altenburger M, Wrbas K. Effectiveness of hand and rotary instrumentation for removing a new synthetic polymer-based root canal obturation material (Epiphany) during retreatment. IntEndod J 2006;39:150-6.

[11]. 11. Nair PNR, Sjögren U, Krey G, Kahnberg K-E, Sundqvist G. Intraradicular bacteria and fungi in root-filled, asymptomatic human teeth with therapy-resistant periapical lesions: a long-term light and electron microscopic follow-up study. J Endod;16:5808 .

[12]. 12. Schirrmeister JF, Wrbas KT, Scheider FH, Altenburger MJ, Hellwig E. Effectiveness of a hand file and three nickel-titanium rotary instruments for removing gutta-percha in curved root during retreatment. Oral Surg oral Med Oral Pathol Oral RadiolEndod 2006;101:542-7.

[13]. 13. Schirrmeister JF, Hermanns P, Meyer KM, Goetz F, Hellwig E. Detectability of residual Epiphany and gutta-percha after root canal retreatment using a dental operating microscope and radiographs: an ex vivo study. IntEndod J 2006;39:558-65.

[14]. 14. De CarvalhoMaciel AC, ZaccaroScelza MF. Efficacy of automated versus hand instrumentation during root canal retreatment: an ex vivo study. IntEndod J 2006;39:779-84.

[15]. 15. Hülsmann M, Stotz S. Efficacy, cleaning ability and safety of different devices for gutta-percha removal in root canal retreatment. IntEndod J 1997;30:227-33.

[16]. 16. Barletta FB, De Mello Radhe N, Limongi O, Maranhao Moura AA, Zanesco C, Mazocatto G. In vitro comparative analysis of 2 mechanical techniques for removing gutta-percha during retreatment. J Cal Dent Assoc 2007;73:65-65e.

[17]. 17. Rhodes JS, Pitt Ford TR, Lynch JA, Liepins PJ, Curtis RV. Micro-computer tomography: a new tool for experimental endodontology. IntEndod J 1999;32:165-70.

[18]. 18. Gu LS, Ling JQ, Wei X, Huang XY. Efficacy of protaper universal rotary retreatment system for gutta-percha removal from root canals IntEndod J 2008;41:288-95.

[19]. 19.Tasdemir T, Er K, Yildirim T, Celik D. Efficacy of three rotary NiTi instruments in removing gutta-percha from root canals. IntEndod J 2008;41:191-6.

[20]. 20. Ruddle CJ. Nonsurgical retreatment. In: Cohen S, Burns RC, eds. Pathways of the pulp (ed 8). St Louis, MO: CV Mosby; 2002:875-930.

[21]. 21. Metzger Z, and Ben-Amar A. Removal of Overextended Gutta-percha Root Canal Fillings in Endodontic Failure Cases. J Endod 1995;21:287-8. 
[22]. 22. McDonald MN, Vire DE. Chloroform in the endodontic operatory. J Endod 1992;18:301-3.

[23]. 23. Wennberg A, Orstavik D. Evaluation of alternatives to chloroform in endodontic practice. Dent Traumatol 1989;5:234-37.

[24]. 24. Saad AY, Al-Hadlaq SM, Al-Katheeri NH. Efficacy of two rotary NiTi instruments in the removal of gutta-percha during root canal retreatment. J Endod 2007;33:38-41.

[25]. 25. Viduèiæ D, Jukiæ S, Karloviæ Z, Bo•iæ •, Miletiæ I, Aniæ I. Removal of gutta-percha from root canals using an Nd:YAG laser. IntEndod J 2003;36:670 -3.

[26]. 26. Baratto-Filho F, Ferreira E, Fariniuk L. Efficiency of the 0.04 taper ProFile during retreatment of gutta-percha filled root canals. IntEndod J 2002;35:651-4.

[27]. 27. Schafer E, Erler M, Dammaschke T. Comparative study on the shaping ability and cleaning efficiency of rotary Mtwo instruments. Part1. Shaping ability in simulated curved root canals.IntEndod J 2006;39:196-202.

[28]. 28.Gergi R, Sabbagh C. Effectiveness of two nickel-titanium rotary instruments and a hand file for removing gutta-percha in severely curved root canals during retreatment: an ex vivo study. IntEndod J. 2007;40:532-37. 